\title{
THE SECULAR RESONANCES IN THE SOLAR SYSTEM
}

\author{
CHRISTIANE FROESCHLE \\ O.C.A.Laboratoire G.D. Cassini, CNRS URA 1362 B.P.229 F-06304 Nice Cedex 4, \\ France \\ E-mail Froesch@obs-nice.fr
}

\author{
ALESSANDRO MORBIDELLI \\ O.C.A.Laboratoire Cerga, CNRS URA 1360 B.P.229 F-06304 Nice Cedex 4, \\ France \\ E-mail Morby@obs-nice.fr
}

\begin{abstract}
In the last three years new studies on secular resonances have been done. The second-order and fourth-degree secular perturbation theory of Milani and Knežević allowed to point out the effect of mean motion resonances on the location of the linear and non linear secular resonances. Moreover this theory improved the knowledge of the exact location of the $g=g_{6}$ (i.e. $\nu_{6}$ ) resonance at low inclination. Morbidelli and Henrard revisited the semi-numerical method of Williams, taking into account the quadratic terms in the perturbing masses. They computed not only the location of secular resonances, but also provided a global description of the resonant dynamics in the main secular resonances namely $g=g_{5}$ (i.e. $\nu_{5}$ ), $g=g_{6}$ (i.e. $\nu_{6}$ ) and $s=s_{6}$ (i.e. $\nu_{16}$ ). The resonant proper element algorithm developed by Morbidelli allows to identify the dynamical nature of resonant objects, and is a powerful tool to study the mechanisms of meteorite transport to the inner Solar System. Purely numerical experiments have been done, which show : (i) the complexity of the dynamics when two resonances overlap; (ii) the efficiency of successive crossings of non linear resonances in pumping up the inclination of small bodies; (iii) the efficiency of the secular resonance $\nu_{6}$ as a source of meteorites up to $2.4 \mathrm{AU}$.
\end{abstract}

\section{Introduction.}

According to secular perturbation theories, the orbital elements of the planets change with periods ranging from thousands to millions of years. If one restricts to the Sun-Jupiter-Saturn system, these changes are quasi-periodic with three basic frequencies : $g_{5}$ (the average precession rate of Jupiter's longitude of perihelion), $g_{6}$ (the average precession rate of Saturn's longitude of perihelion) and $s_{6}$ (the precession rate of both nodes); additional frequencies appear when the full solar system is taken into account. The planets exert also secular perturbations on any small body orbiting around the Sun and force the precession of their orbits; we denote by $g$ the precession frequency of the asteroid's longitude of perihelion, and $s$ the precession frequency of its node. These secular perturbations give particularly large effects when a secular resonance occurs, namely when the frequency of precession of the small body $g$ or $s$ (or a combination of these frequencies) becomes nearly equal to an eigenfrequency (or a combination of eigenfrequencies) of the planetary system. In the following we will call linear secular resonances those involving only one asteroid frequency and one planetary frequency.

In the present review (since previous ones have been published, Scholl et al. 1989, Froeschlé and Scholl 1989) we will just mention the most important steps which 
have been done in the field of secular resonances up to 1989 and we will devote this paper to the new results obtained after 1989.

The phenomena of secular resonances have been known since the end of the last century (LeVerrier 1855, Tisserand 1882, Poincaré 1892). The first modern approach to study secular resonances was done by Williams (1969). He developed a semi-numerical theory based on Gauss averaging method, which can be applied to derive proper elements of asteroidal orbits even for large values of eccentricity and/or inclinations, and mapped the contours of secular resonances in the màin asteroid belt as a function of the proper elements $a, e$ and $i$. This method was later refined (Williams and Faulkner 1981) to derive detailed graphical maps of the surfaces which denote the location of the three strongest secular resonances, in the proper element space with semi major axis ranging between 1.25 and $3.5 \mathrm{AU}$.

Since the development of a new generation of high speed computers, orbital evolution of resonant and near resonant asteroids have been studied. These purely numerical experiments were based mainly on the four body model, i.e. Sun-JupiterSaturn-Asteroid (see Froeschlé and Scholl 1989). The numerical experiments yielded new quantitative results and confirmed the qualitative behaviour of orbital evolution at secular resonances obtained previously by semi-analytical theories. Moreover they revealed the existence of chaotic motions.

These numerical works revived the interest for the study of secular resonances and, as a consequence, new improved theories appeared such as the ones by Yoshikawa (1987) and Šidlichovský (1989), for the $g=g_{6}$ resonance, and by Nakai and Kinoshita (1985), for the $s=s_{6}$ resonance (see Froeschlé and Scholl 1989 for a review of these works). The more recent theories were developed by Milani and Knežević (1990), and also by Morbidelli and Henrard (1991a).

Milani and Knežević (1990) developed an analytical theory to compute proper elements, where only Jupiter and Saturn are considered. Their work is based on an explicit expansion of the hamiltonian of the model in powers of the eccentricity and inclination of both the asteroid and the planets; such expansion is truncated at degree 4 for what concerns the part which is linear in the masses of the planets, and degree 2 for what concerns the quadratic part. All the terms of order larger than 2 in the masses are also neglected. As a result they located in the proper element space not only the linear secular resonances but also the ones of degree 4 in eccentricity and inclination. Retaining the quadratic terms in the perturbing masses they pointed out the strong effects imposed by mean motion resonances on the location of secular ones. Knežević et al.(1991), generalizing the previous theory by considering the perturbation of the four major planets, determined the location of the linear secular resonances from 2 to 50 AU. Milani and Knežević (1992) published an improved version of their theory, taking into account the secular perturbation of the four outer planets and part of the effect of the inner planets. They studied the effects of non linear secular resonances upon asteroid families. Finally, Milani and Knežević (1994) analyzed in detail the dynamical behaviour of several asteroids close to some non linear secular resonances.

Šidlichovský (1990) extending his non linear theory developed in 1989 studied the problem of overlapping of secular resonances. The simple Chirikov overlapping criterion was applied to the hamiltonian where both terms corresponding to secular 
resonances $g=g_{5}$ and $g=g_{6}$ are taken into account. The overlap criterion yields a critical value of the quantity $P=\sqrt{\left(1-e^{2}\right)} \cdot(1-\cos i)$ depending on the semimajor axis of the asteroid. When $P$ is greater than the critical value, resonance overlap occurs and chaotic motion appears. A mapping of the hamiltonian is presented which allows to calculate rapidly trajectories.

All these theories, based on the classical expansion of the perturbation in power series of the asteroid's eccentricity and inclination, have necessary a poor accuracy in determining the location and the dynamics of secular resonances at large eccentricity and inclination. Therefore, Morbidelli and Henrard (1991a) revisited the semi-numerical method first developed by Williams (1969), avoiding the expansion in power series of asteroid's $e$ and $i$. Introducing suitable action-angle variables, they take completely into account the strongly non-linear dynamics related to the motion of the perihelion argument of the small body, which is dominant at high inclination, as shown by Kozai (1962). Furthermore they improved Williams' results on the location of resonances retaining the quadratic terms in the mass ratio (neglected by Williams). Moreover they studied in detail the dynamics of the three principal secular resonances $g=g_{6}$ (i.e. $\nu_{6}$ in Williams' notations), $g=g_{5}$ (i.e. $\nu_{5}$ ) and $s=s_{6}$ (i.e. $\nu_{16}$ ) (Morbidelli and Henrard, 1991b).

Recently Morbidelli (1993) developed an efficient algorithm for the computation of the dynamical evolution of asteroids which are inside or close to a secular resonance. This algorithm is able to identify the dynamical nature of resonant objects. Since it requires a short CPU time for its execution, it is a powerful tool to study the dynamics of many fictitious objects in order to investigate the mechanisms of meteorite transport to the Earth. In addition, this work has pointed out the dynamical peculiarities of the $g=g_{6}$ resonance.

In the last years new numerical experiments have been performed (Scholl and Froeschlé 1991, Froeschlé and Scholl 1992, Farinella et al. 1993a) including not only Jupiter and Saturn but also Mars and the Earth. These new results show the role played by the secular resonances -linear and non linear ones- in the distribution of the asteroids in the inner belt and also the importance of the secular resonance $g=g_{6}$ for the delivery of meteorites to the Earth.

In the following we review in Section 2 the new theories developed since 1990. Then we discuss the new results obtained by numerical experiments in section 3 .

\section{Theories.}

The recent works on secular resonances can be classified in two groups : the analytical theory which consists in expanding the perturbation with respect to the eccentricity and the inclination of the small body, and the semi-numerical one which avoids any expansion in series of the small body's $e$ and $i$, and therefore require the numerical evaluation of integrals.

\section{1. MILANI- KNEŽEVIĆ APPROACH (MK).}

In order to compute proper elements (i.e. quasi-integrals of motion) the MK theory was first developed in 1990, and improved in 1992 and 1993 by adding the new 
perturbation terms; it is based on the Lie series formalism and uses an iterative algorithm. In the disturbing function which is averaged to eliminate short periodic terms, they include the first order term in the masses expanded up to the degree 4 in eccentricity and inclination (both for the asteroid and the planets), and the quadratic term in the masses expanded up to the second degree. Their results on the location of secular resonances are a byproduct of their proper elements work.

Indeed secular resonances enter the proper elements theory as small divisors which contain combinations of the fundamental frequencies $g$ and $s$ of the asteroid and the fixed frequencies $g_{j}$ and $s_{j}$ of the planets. Therefore, in order to compute the location of secular resonances one has to compute the values of the frequencies $g$ and $s$ as functions of the proper orbital elements and search for those that satisfy a resonance condition; in particular linear secular resonances (the strongest ones) are given by the relations $g=g_{j}$ and $s=s_{j}$. As it is well known, classical linear theories provide two basic frequencies $g=g_{\circ}$ and $s=s_{\circ}$, with $s_{\circ}=-g_{\circ}$, functions of the semi-major axis only; $g_{\circ}$ and $s_{\circ}$ are also called "free oscillation frequencies". In Milani and Knežević work, $g$ and $s$ are given by $g_{\circ}$ and $s_{\circ}$ corrected by considering the contribution of the quadratic term in the masses (which makes $s_{\circ} \neq-g_{\circ}$ ) plus a correction coming from the terms of degree 4 in $e$ and $i$; this makes the final frequencies to become functions also of the proper eccentricity and inclination.

Milani and Knežević work points out the effect of mean motion resonances on the location of secular ones. This is due to the fact that the frequencies are corrected by the quadratic term in the masses which contains small denominators corresponding to the main mean motion resonances. On the other hand, since the quadratic term in the masses is computed up to degree 2 only in $e$ and $i$, only the effect of mean motion resonances of the form $p+1: p$ and $p+2: p$ is detected.

An example of their results is reported in Figure 1 where the location of secular resonances is plotted in the proper elements space $(a, i)$ for $e=0.1$.

This work has been generalized by Knežević et al. (1991) who determined the location of the linear secular resonances up to the outer solar system. The basic result is that none of the considered secular resonances exist beyond $50 \mathrm{AU}$, so that one can conclude that these secular resonances are not effective for transporting inwards comets belonging to a possible Kuiper belt.

For what concerns the study of the resonant dynamics, Milani and Knežević (1992) have done a detailed study only on the effects of the resonances $g+s=g_{6}+s_{6}$ and $g+s=g_{5}+s_{7}$, which both cut the Eos family. Even if these resonances have a minor effect on the capability to identify the family members, they strongly influence the long term dynamics of Eos family. Also the small Lydia family is strongly perturbed by these resonances. As it is underlined by the authors, the effects of non linear secular resonances on a small family can result in the loss of some family members and even in the impossibility of identifying the family in a reliable way.

More recently Milani and Knežević (1994) analyzed the dynamical behaviour of several asteroids close to non-linear secular resonances, by numerical integrating their orbits for several million years and by computing adaptive proper elements (i.e. proper elements computed by dropping the resonant term) as function of time. The asteroids they have investigated are the following ones : 8 Flora $\left(3 g+s=3 g_{6}+\right.$ 


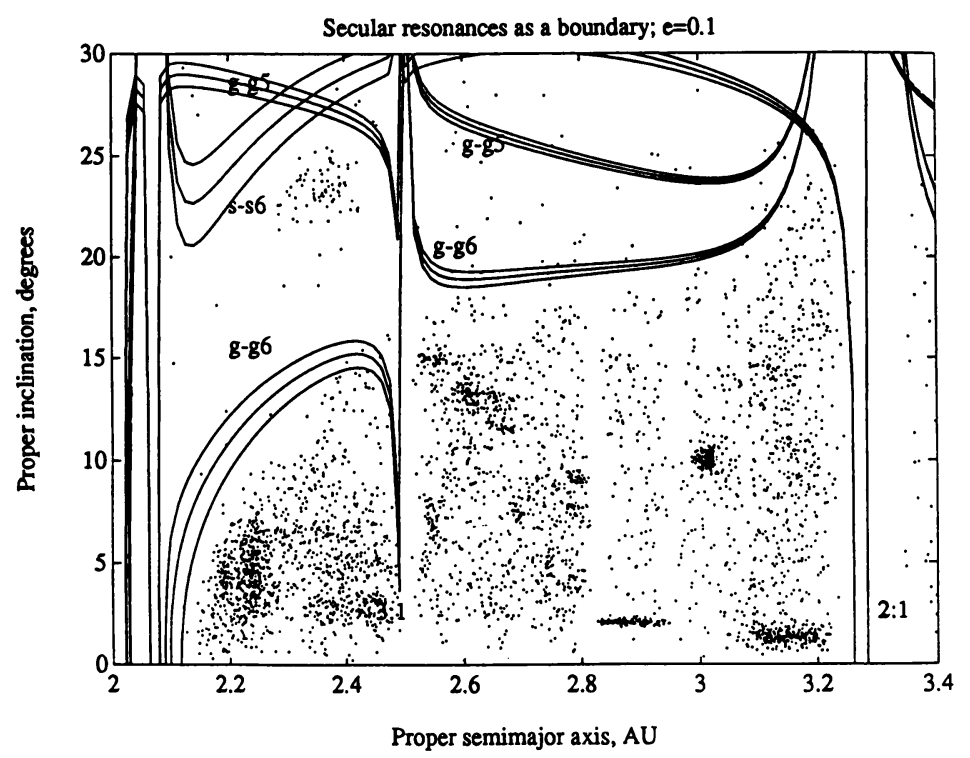

Fig. 1. The location of the main linear secular resonances for proper $e=0.1$ according to Milani and Knežević (1990). The location of the resonances is strongly affected by the interaction with the mean motion resonances $3 / 1$ and $2 / 1$. The contour lines around the secular resonances correspond to a width of $+/-1$ " $/ \mathrm{yr}$.

$\left.s_{6}\right) ; 1047$ Geisha and $244 \mathrm{Sita}\left(2 g+s=2 g_{6}+s_{6}\right) ; 2649$ Oongaq $\left(s=g_{5}-g_{6}+s_{6}\right) ; 59$ Elpis, 244 Oceana and 214 Aschea $\left(g=2 g_{6}-g_{5}\right)$; 1809 Prometheus $\left(g=3 g_{6}-2 g_{5}\right)$.

The main limit of Milani and Knežević work is due to their perturbation expansion. First of all, since the hamiltonian is expanded in power series of the asteroid's eccentricity and inclination, their results loose accuracy at large $e$ or $i$. Moreover, since the term $e^{2} i^{2} \cos (2 \omega)$ is considered only as a "perturbation", the region at large inclination cannot be studied properly, since this term (as pointed out by Kozai) becomes dominant, giving a strongly non-linear dynamics. Nevertheless their results have a good accuracy for the low to moderate $e$ and $i$.

\section{2. MORBIDELLI AND HENRARD APPROACH (MH).}

The MH theory -close to that of Williams (1969)- avoids any truncation of powers of the asteroid's eccentricity and inclination. The averaged hamiltonian -as in Williams' method- is expanded in power series of the planetary eccentricities and inclinations,(i.e. $e^{\prime}$ and $i^{\prime}$ are assumed implicitly to be smaller than whatever the asteroid's $e$ and $i$ ). The quadratic terms in the perturbing masses (which were completely neglected in Williams' work) are taken into account.

Introducing suitable angle-action variables their method can be applied all over the phase space, i.e. in regions of circulation and also libration of the perihelion argument. In order to study the dynamics of the secular resonances, Morbidelli and Henrard develop a local perturbation study in the neighbourhood of a resonant 
orbit. Moreover, they deduce from the local study suitable integrable global models for the description of the dynamics of the three main secular resonances $g=g_{5}$, $g=g_{6}$ and $s=s_{6}$.

The main steps for what concerns the localization of secular resonances are the following (Morbidelli and Henrard, 1991a) :

- The hamiltonian is split into an integrable part (hamiltonian of the 2-body problem) and a perturbation one. The perturbation part is globally of order Jupiter mass $\sim 10^{-3}$, and is time-dependent through the planetary elements which are considered as given functions of time with fixed proper frequencies $g_{j}$ and $s_{j}$.

- the hamiltonian is averaged with respect to short periodic terms by applying the Lie algorithm and truncated at order two in the masses. The linear term in the masses is computed without any expansion in powers of $e$ and $i$; conversely the quadratic term in the masses is computed up to degree 4 only. More recently, Lemaitre and Morbidelli (1994) computed the quadratic term in the masses in a semi-numerical way, i.e. avoiding expansions.

- the averaged hamiltonian is expanded in power series of $e^{\prime}$ and $i^{\prime}$, which are assumed to be the new perturbation parameters, denoting $K_{m}$ the term of degree $m$ in $e^{\prime}, i^{\prime}$. Therefore, the main part of the averaged hamiltonian turns out to be the one given by the planets assumed on coplanar circular orbits, called $K_{0}$; this part is integrable, although highly non-linear, and describes completely the dynamics related to the motion of the argument of perihelion, which is dominant at large inclination, as pointed out by Kozai (1962). In particular at large inclination, the argument of perihelion may librate, locked in the "Kozai resonance".

- Suitable action-angle variables are introduced in order to eliminate the dependence of $K_{0}$ on the argument of perihelion. In the new variables, $K_{0}$ is characterized by two fixed frequencies, which are the proper frequencies $g$ and $s$ of the system. The comparison of these frequencies with those of the planetary system gives the location of secular resonances. If the quadratic term in the masses is neglected, Morbidelli and Henrard recover completely the result of Williams and Faulkner (1981). The inclusion of the quadratic terms, confirms the results of Milani and Knežević on the influence of mean motion resonances on the location of secular ones. However, the authors pointed out that the results depend critically on the degree of truncation of the computation of the quadratic term, especially close to the $2 / 1$ mean motion commensurability $(3.0-3.2$ A.U.) (see Figure 2.). Recently Morbidelli et al. (1993b), applying the method of successive elimination of harmonics, have computed the proper frequencies $g$ and $s$ in this region without passing through the computation of higher order terms in the masses, therefore obtaining much more reliable results (see Fig. 3).

For what concerns the description of the resonant dynamics, Morbidelli and Henrard's work (1991b) provides a global description of the motion in the main linear resonances $g=g_{6}, s=s_{6}$, and partly also in $g=g_{5}$.

Basically, the linear term in $e^{\prime}, i^{\prime}$ (called $K_{1}$ ) is written in the action-angle variables introduced in order to normalize $K_{0}$; furthermore, assuming to be in presence of only one isolated resonance, only the corresponding resonant term is 

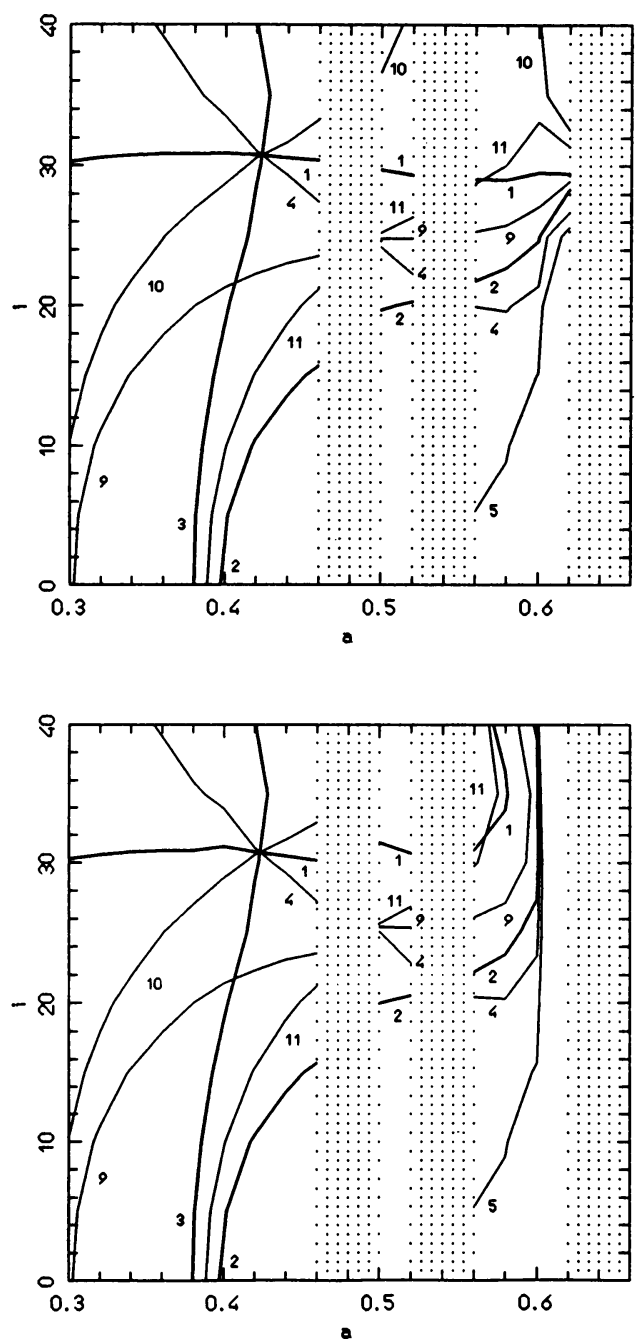

Fig. 2. The location of secular resonances for proper $\epsilon=0.1$ according to Morbidelli and Henrard (1991a). The semi major axis is expressed in units of Jupiter ( $a_{J}=5.2026$ A.U.) The enumeration of the resonances is the following : $1 g=g_{5} ; 2 g=g_{6} ; 3 s=s_{6}$; $4 g=s=g_{5}+s_{6} ; \mathbf{5} g+s=g_{6}+s_{6} ; \mathbf{9} 2 g=g_{5}+g_{6} ; \mathbf{1 0} g-s=g_{5}-s_{6} ; \mathbf{1 1}$ $g-s=g_{6}-s_{6}$. In the picture above the quadratic term in the masses is truncated at order 2 , in the picture below at order 4 . The comparison shows how such a computation is sensitive to the truncation order, at least in the region at large inclination, in between 2.9 and 3.2 AU. The dotted bands cover the mean motion commensurabilities $3 / 1,5 / 2$ and $2 / 1$ which are forbidden regions for the theory since the computation of the quadratic term of the masses is singular. 


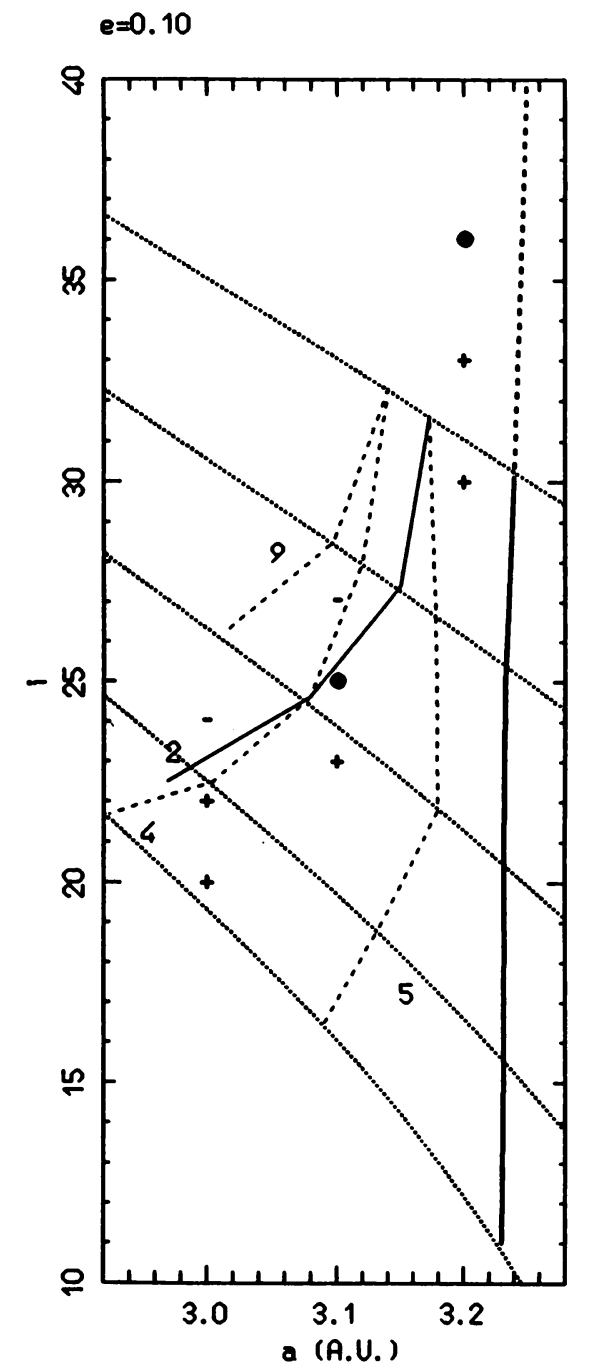

Fig. 3. The location of secular resonances at proper $e=0.1$ and $a$ between 2.9 and 3.3 AU according to the more sophisticated theory by Morbidelli et al. (1993b). The enumeration of the resonances is the same as in figure 2. The vertical line at $3.24 \mathrm{AU}$ denotes the separatrix of the $2 / 1$ mean motion commensurability. Secular resonances are bent to larger inclination approaching the $2 / 1$ commensurability. This is confirmed by numerical integrations : the + denotes the initial conditions of test orbits which turn out to be below the $g=g_{6}$ secular resonance (2); the - denotes those which are above the resonance; the $\otimes$ denotes those which are inside the secular resonance. 
retained in $K_{1}$, while the other terms are dropped. This gives an integrable model (similar to the one usually called "second fundamental model", see Henrard and Lemaitre, 1983) of the considered resonant dynamics. The main results can be shortly summarized as follows :

- $g=g_{6}$ : the global pictures of the resonant phase space are only in partial agreement with those of the previous work by Yoshikawa (1987), but are confirmed by numerical simulations;

- $s=s_{6}:$ the results confirm the previous ones by Nakai and Kinoshita (1985), in the region at moderate inclination where $\omega$ (perihelion argument) circulates. Moreover the $\nu_{16}$ resonance is found also in the region of $\omega$-libration. In Froeschle et al. (1991), the theory is applied in order to give a complete explanation of the orbital behaviour of the asteroid 2335 James;

- $g=g_{5}$ : this resonance is located near a critical threshold where the coefficient of the corresponding resonant term in $K_{1}$ changes the sign; therefore the uncertainty on the global resonant picture is great, due mainly to the poor accuracy of the quadratic term in the masses computed, at this step, from truncated power series of $e$ and $i$.

\section{3. QUANTITATIVE RESONANT DYNAMICS.}

The MH theory on the dynamics in the main secular resonances has been recently improved from the quantitative point of view and implemented in the resonant proper elements program by Morbidelli (1993). The basic approach is the following:

1) the perturbation term $K_{1}$ is expanded in Fourier series of the secular angles;

2) all the non-resonant terms in $K_{1}$ are eliminated via a canonical transformation similar to that used for the computation of usual proper elements; this gives the "semi-proper elements" of the asteroid;

3 ) in the semi-proper elements space, the hamiltonian is thus reduced to an integrable resonant one of the kind $K_{0}+k_{1} \cos \sigma$, where $\sigma$ is the critical angle of the secular resonance (for example $\varpi-g_{6} t$ for the $g=g_{6}$ resonance), and $k_{1}$ is the coefficient of the resonant term in the Fourier expansion of $K_{1}$;

4) the semi-proper elements of the asteroid are the initial conditions for the integration of such resonant hamiltonian : the solution gives the long term dynamical evolution of the considered body. In particular the resonant proper elements are defined as the values of e and i while $\sigma=0^{\circ}, 180^{\circ}$.

Figure 4 shows an example of application of the resonant proper elements algorithm on a fictitious asteroid in the $g=g_{6}$ resonance. On the left hand side the global picture of the resonant phase space is plotted, computed as level curves of $K_{0}+k_{1} \cos \sigma$; the dot marks the actual semi-proper elements of the asteroid, i.e. the present position of the asteroid on such a phase space. On the right hand side, the full numerical integration of the secular behaviour of the object is plotted, for a check. The coordinates are $x=e \cos \sigma$ and $y=e \sin \sigma$. As one sees, the resonant proper elements algorithm describes accurately the long term dynamical evolution of the object, averaging out all the "short" periodic oscillations due to the nonresonant secular terms. Additional examples, referring also to the case where the eccentricity blows up, can be found in Morbidelli (1993). 

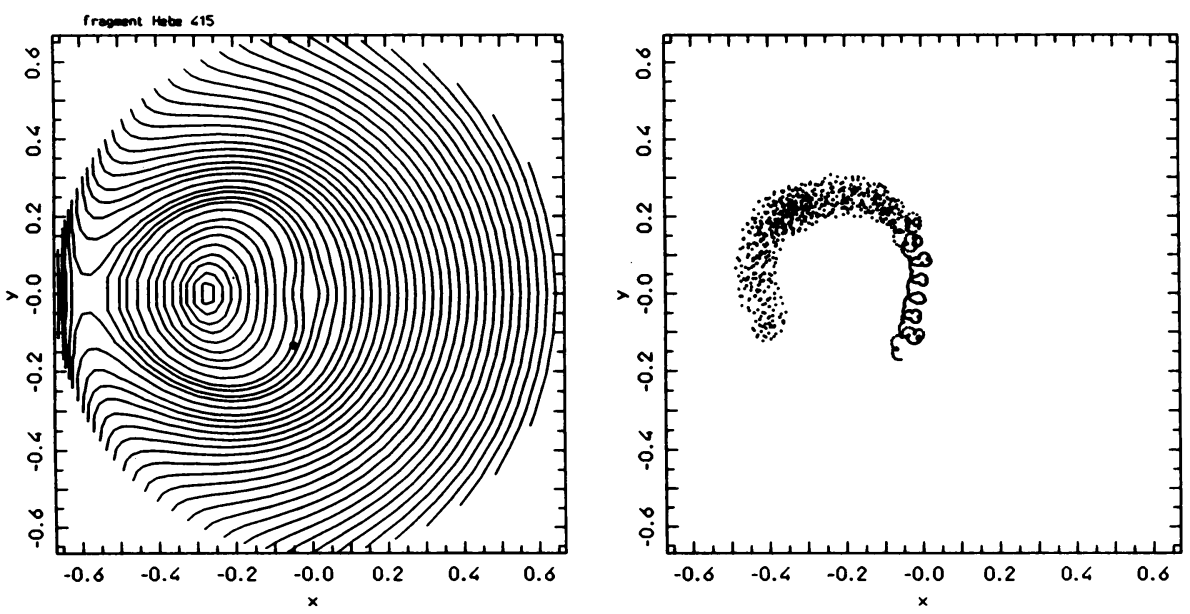

Fig. 4. On the left, the phase space computed by the resonant proper element algorithm for a fictitious asteroid. The dot marks the present position of the body in the semi-proper elements space. On the right, the evolution of the orbit of the body as integrated numerically. The coordinates are $x=e \cos \sigma$ and $y=e \sin \sigma$, where $\sigma$ is the critical angle of the $g=g_{6}$ secular resonance.

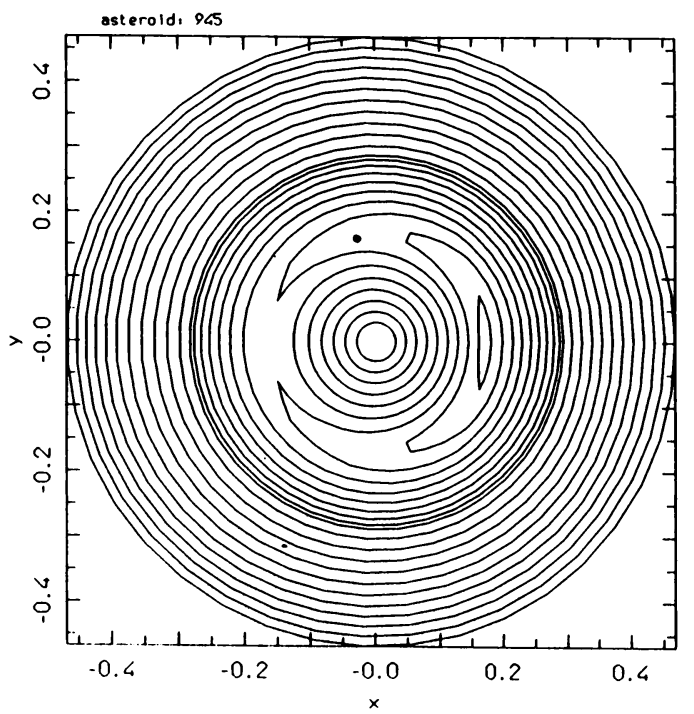

Fig. 5. The phase space of the $g=g_{5}$ resonance for the asteroid 945 Barcelona. The dot marks the present position of the asteroid in the semi-proper elements space. 

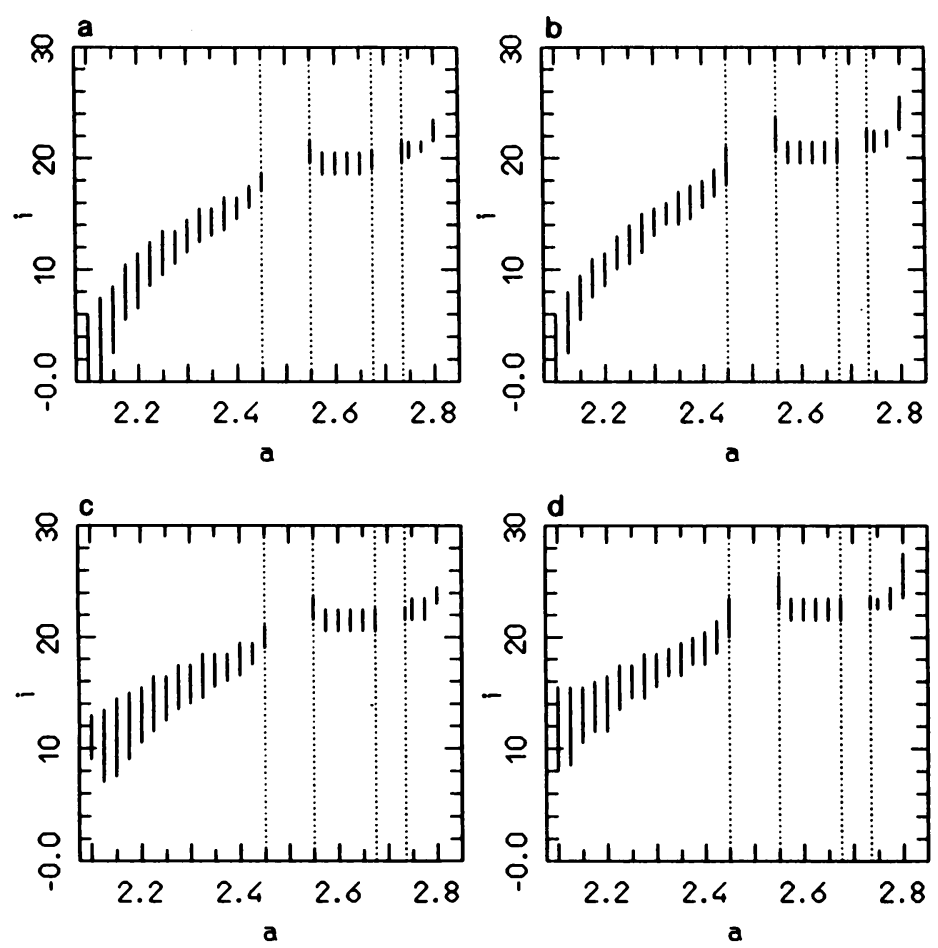

Fig. 6. The sets of the initial conditions in the $g=g_{6}$ resonance with $e=0.15$, which lead to $e>0.4$. Figure a (top left) is made for $\omega=0^{\circ}$ and $\Omega=124.19^{\circ}\left(\sigma=0^{\circ}\right)$; figure b (top right) is for $\omega=180^{\circ}$ and $\Omega=124.19^{\circ}\left(\sigma=180^{\circ}\right)$; figure c (bottom left) is for $\omega=180^{\circ}$ and $\Omega=304.19^{\circ}\left(\sigma=0^{\circ}\right)$; figure d (bottom right) is for $\omega=0^{\circ}$

The quantitative improvement with respect to the original $\mathrm{MH}$ theory on secular resonant dynamics is due to two facts :

$\mathrm{a}$ - instead of neglecting all non-resonant terms in $K_{1}$, one eliminates them via the suitable variable transformation which gives the semi-proper elements;

$\mathrm{b}$ - the quadratic term in the masses is taken into account and computed in a seminumerical way which allows to avoid expansion in powers of the asteroid's eccentricity and inclination.

As a consequence, the resonant proper elements algorithm can be applied with reliability also to the $g=g_{5}$ resonance (see fig. 5 for the asteroid 945 Barcelona).

The relevant advantage of the resonant proper elements algorithm with respect to pure numerical integration is the CPU cost : to construct a global picture of the phase-space as in figures 4 and 5 (which corresponds to computing the dynamical evolution for millions of years) takes less than 1 minute of CPU time on a HP710. This allows, on the one hand, to study thousands of real/fictitious bodies, as in Morbidelli et al. (1993a) for the investigation of dynamical mechanism of meteorite 
transport; on the other hand, a systematic exploration of the resonant dynamics, by analyzing hundreds of initial conditions chosen on a suitable grid. This allowed, for example, to point out the dynamical peculiarities of the $g=g_{6}$ resonance, which is the only secular one able to pump up the asteroid's eccentricity up to extremely large values $(e>0.8)$. Figure 6 shows the sets of initial conditions in the $(a, i)$ mean elements space with $e=0.15$ and different values of the angles, which lead to $e>0.4$; in other words, the dangerous regions of the $g=g_{6}$ resonance.

The main limit of the resonant proper elements algorithm is that it does not take into account the inner planets; as a consequence close approaches, which are the relevant dynamical features of any orbit with large eccentricity, are not taken into account in the model. Therefore, one can apply the algorithm in order to study the dynamical evolution of an object only up to the time when the first close approach occurs.

\section{Numerical Experiments.}

As pointed out in the introduction, a lot of numerical experiments have been done since the 1980's. Most of them investigated the orbital evolution of fictitious and real asteroids located in or near the principal resonances. These quantitative experiments gave a detailed picture of the dynamical properties of the secular resonances. Transitions between libration, inner and outer circulations have been found, indicating the occurrence of chaotic motion.

Froeschle et al. (1991) have pointed out the complex dynamical behaviour of the asteroid 2335 James (first found located by Williams in the $s=s_{6}$ resonance). A backwards integration showed that James was temporarily located in the $g=g_{5}$ and $s=s_{6}$ resonances. In a Sun-Mars-Jupiter-Saturn model, the numerical integration showed close encounters of the asteroid with Mars, without ejecting James from the $s=s_{6}$ resonance.

Numerical experiments on fictitious small bodies with initial eccentricities e $=0.1$ have been performed in the overlapping region of the $3 / 1$ mean motion resonance and of the $g=g_{6}$ secular resonance (Froeschlé and Scholl 1993). The dynamics in this region is very complex as suggested by the recent theories. The analysis of the dynamical behaviour of orbits in the $g=g_{6}$ secular resonance shows that the dynamical picture of the phase space changes approaching the $3 / 1$ mean motion commensurability : for example, the libration motion is reversed. Inside the $3 / 1$ resonance region the secular resonance $g=g_{5}$ is the dominant one, and some secondary secular resonances as $g-s=g_{6}-s_{6}$ and $2 g=g_{5}+g_{6}$ are present.

Froeschlé and Scholl (1992) studied the effects of linear and non-linear secular resonances in the inner belt $a \leq 2.4 \mathrm{AU}$, which appears to be depopulated at inclinations larger than $12^{\circ}$. This region is surrounded by the three main resonances $g=g_{5}, g=g_{6}, s=s_{6}$ and is crossed by non-linear ones. Numerical experiments of fictitious bodies show that the inclinations are pumped up by successive crossings through the non linear resonances. Bodies located at the border of the $g=g_{6}$ resonance with semi major axis $a \leq 2.4$ AU become Earth-crossers on a time scales of 1 Myr.

Many studies have shown the potential importance of secular resonances for the delivery of meteorites and Aten/Apollo/Amor objects to planet-crossing orbits, 

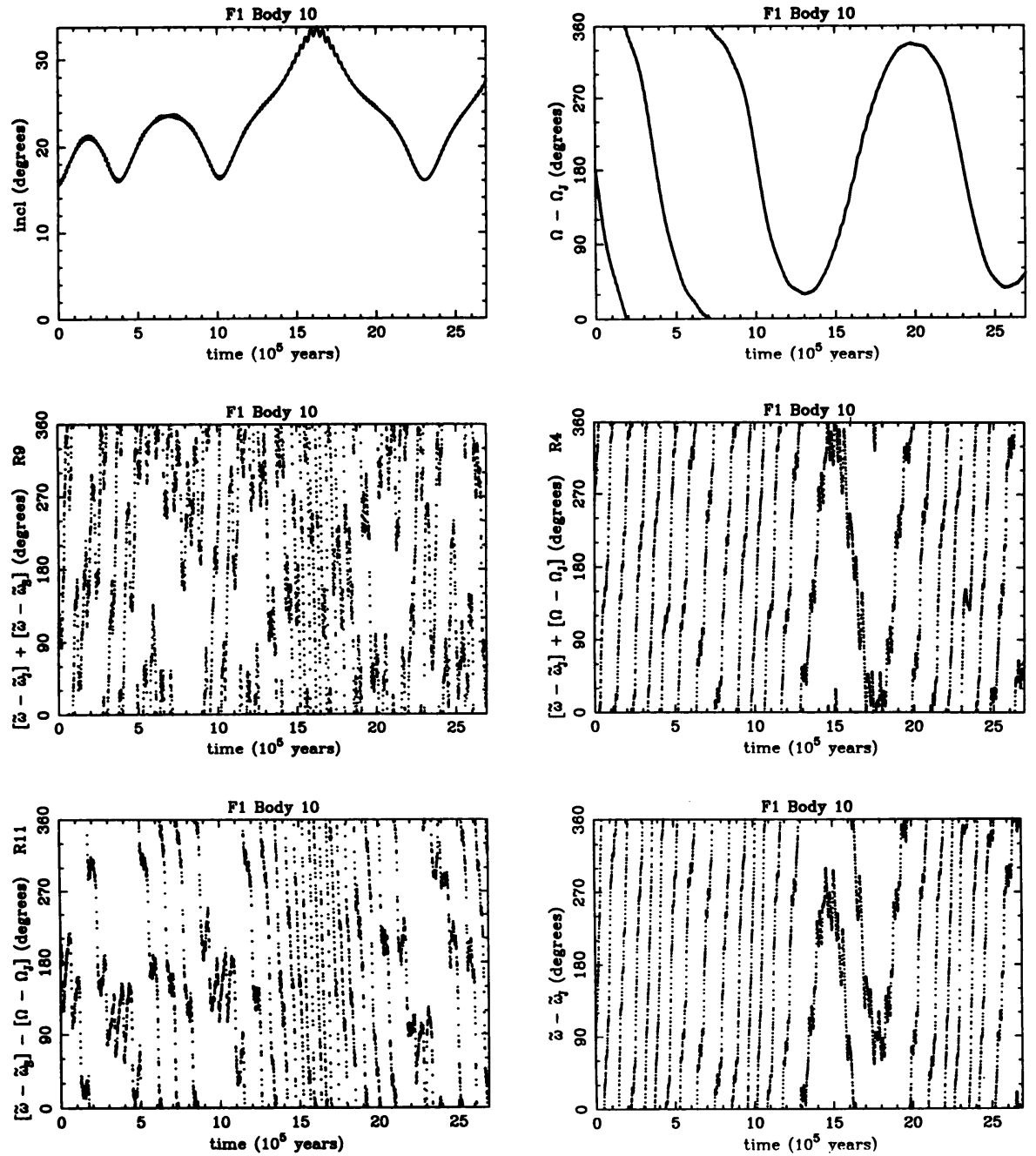

Fig. 7. An unpublished numerical simulation by Froeschlé and Scholl (see also Froeschlé and Scholl, 1992) which shows a fictitious body the inclination of which is pumped up from 16 to 34 degrees by chaotic diffusion through secular resonances. The orbit first crosses the secular resonances $2 g=g_{5}+g_{6}$ (critical argument $\tilde{\omega}-\tilde{\omega}_{J}+\tilde{\omega}-\tilde{\omega}_{S}$ ) and $g-s=g_{6}-s_{6}$ (critical argument $\tilde{\omega}-\tilde{\omega}_{S}+\Omega-\Omega_{J}$ ) and is finally captured in the resonance $s=s_{6}$ (critical argument $\Omega-\Omega_{J}$ ) and, temporary, in the $g=g_{5}$ (critical argument $\left.\tilde{\omega}-\tilde{\omega}_{J}\right)$. 
often using Monte-Carlo techniques to model the chaotic orbital evolution of asteroid fragments (Wetherill 1977, 1979, 1988, Wetherill and Williams 1979). Milani et al. (1989) suggested, on the basis of a numerical investigation, that the region of secular resonances around $2 \mathrm{AU}$ could be at least as important as the Kirkwood gaps as a route for Earth crossing.

Scholl and Froeschlé (1991) showed that the proximity between the $g=g_{6}$ secular resonance and the $4 / 1$ mean motion resonance broadens significantly the Earth-crossing region in the inner belt. In the Sun-Mars-Jupiter-Saturn model, integrations of bodies with initial eccentricity equal to 0.05 and 0.1 have been performed for several semimajor axes $a \leq 2.13 \mathrm{AU}$. Due to the proximity of the resonance $g=g_{6}$ the eccentricity is pumped up inducing close encounters with Mars. At that time random walk of the semimajor axis starts and the body can be trapped either in the resonance $4 / 1$, which makes the eccentricity to increase up to 0.5 after $10^{4}$ years, or in the $g=g_{6}$ resonance; in the latter case the time scale to become Earth-crosser is at least $10^{5}$ years.

Farinella et al. 1993a have quantitatively modeled the chance insertion of collisional fragments into the $g=g_{6}$ and $3 / 1$ resonances, through which they can achieve Earth-crossing orbits. They show that the two resonances are potentially effective channels for fragment collection and delivery. The efficiency of the $g=g_{6}$ is comparable to the efficiency of the $3 / 1$ resonance. Moreover the $g=g_{6}$ resonance is found to be an efficient fragment collector not only near the inner edge of the asteroid belt, but also for several asteroids with semimajor axes about 2.4 and 2.7 $\mathrm{AU}$, see Farinella et al., this volume.

Numerical integrations of 18 fictitious fragments ejected from the asteroid 6 Hebe located very close to the $g=g_{6}$ resonance at semimajor axe of 2.42 AU have been performed by Farinella et al. 1993b. For five of these fragments the authors found that the resonance $g=g_{6}$ pumps up the eccentricity to value $\geq 0.6$ which result into Earth-crossing within a time scale of $1 \mathrm{Myr}$, several close encounters with our planet cause chaotic orbital evolution. Some more fragments become Mars-crossers albeit not Earth-crossers. Two bodies are injected into the $3 / 1$ mean motion resonance with Jupiter, and display also chaotic behaviour leading to Earth-crossing.

\section{The new frontiers}

The many numerical and theoretical works which have been done since the beginning of this decade have thrown a new light onto the secular evolution of the orbits in the main asteroid belt pointing out the relevant dynamical role of secular resonances.

However, some problems of non-negligible importance for a deeper comprehension of the features of the solar system, are still to be investigated.

First of all, we stress that most of the works described above are devoted to isolated secular resonances. Just few papers (Šidlichovský, 1990; Froeschlé and Scholl, 1992, Milani and Knežević, 1994) point out the importance of interactions among secular resonances. In particular, overlapping among non-linear secular resonances could give rise to important phenomena of diffusion through the asteroid belt; this is the way by which faint resonances could induce macroscopic phenomena. Conversely, isolated secondary resonances could give rise to chaotic phenomena (with 
positive Liapunov exponent) but with no importance on macroscopic stability, in the sense that the action variables do not show significant changes.

Moreover, the secular theories and the numerical experiments should be extended to the outer solar system, following the road opened by Knežević et al. (1991) who, on the other hand, did not provide any information on the strength of secular resonances and, consequently, on their dynamical role.

A new subject of work is the role of secular resonances inside mean motion commensurabilities. A recent paper by Morbidelli and Moons (1993) shows that secular resonances exist and are responsible of the existence of large chaotic zones. This seems to be a promising way to explain the existence of Kirkwood gaps.

\section{References}

Farinella, P., R. Gonczi, Ch Froeschlé and Cl. Froeschlé 1993a. The Injection of Asteroids Fragments into Resonances. Icarus 101, 174-197.

Farinella, P., Ch. Froeschlé and R. Gonczi 1993b. Meteorites from the asteroid 6 Hebe. Celest. Mech. 56,287-305.

Froeschlé, Ch., A. Morbidelli and H. Scholl 1991. Complex Dynamical Behaviour of The Asteroid 2335 James associated with the Secular Resonances $\nu_{5}$ and $\nu_{16}$ : Numerical Studies and Theoretical Interpretation. Astron. Astrophys. 24, 553-562.

Froeschlé, Ch. and H. Scholl 1989. The three principal secular resonances $\nu_{5} \nu_{6}$ and $\nu_{16}$ in the asteroidal belt. Celest. Mech. 46, 231-251.

Froeschlé, Ch. and H. Scholl 1992. The Effect of Secular Resonances in the Asteroid Region between 2.1 and 2.4 AU. Asteroids Comets and Meteors 1991, 205-209.

Froeschlé, Ch. and H. Scholl 1993. Numerical experiments in the $3 / 1$ and $\nu_{6}$ overlapping region. Celest. Mech. 56, 163-176.

Henrard, J., and A. Lemaitre 1983. A second fundamental model for resonance. Celest. Mech. 30, 197-218.

Knežević, Z., A. Milani, P. Farinella, Ch. Froeschlé and Cl. Froeschlé 1991. Secular Resonances from 2 to $50 \mathrm{AU}$. Icarus $93,316-330$.

Kozai, Y. 1962. Secular perturbations of asteroids with high inclination and eccentricities. Astron. J. 67, 591-598.

Lemaitre, A. and A. Morbidelli 1994. Calculation of proper elements for high inclined asteroidal orbits. Celest. Mech., in press.

LeVerrier, U. J. 1855. Développement de la fonction qui sert de base au calcul des mouvements des planètes. Ann. Obs. Paris 1, 258-342.

Milani, A., M. Carpino, G. Hahn and A. M. Nobili 1989. Dynamics of planet-crossing asteroids : classes of orbital behavior. Project SPACEGUARD. Icarus 78, 212-269.

Milani, A. and Z. Knežević 1990. Secular perturbation theory and computation of asteroid proper elements. Celestial Mechanics 49, 247-411.

Milani, A. and Z. Kneževic̀ 1992. Asteroid proper elements and secular resonances. Icarus 98, 211-232.

Milani, A. and Z. Kneževič 1994. Asteroid proper elements and the dynamical structure of the asteroid main belt. Icarus, in press.

Morbidelli, A. and J. Henrard 1991a. Secular resonances in the asteroid belt : Theoretical perturbation approach and the problem of their location. Celest. Mech. 51, 131-168.

Morbidelli, A. and J. Henrard 1991b. The main secular resonances $\nu_{5}, \nu_{6}$ and $\nu_{16}$ in the asteroid belt. Celest. Mech. 51, 169-198.

Morbidelli, A. 1993. Asteroid secular resonant proper elements. Icarus 105, 48-66.

Morbidelli, A. and M. Moons 1993. Secular resonances in mean motion commensurabilities : the $2 / 1$ and $3 / 2$ cases. Icarus 102, 316-332. 
Morbidelli, A., R. Gonczi, Ch. Froeschlé, and P. Farinella 1993a. Delivery of meteorites through the $\nu_{6}$ secular resonance. Astron. Astrophys., in press.

Morbidelli, A., H. Scholl and Ch. Froeschlé 1993b. The location of secular resonances close to the $2 / 1$ commensurability. Astron. Astrophys. 278, 644,653.

Nakai,H., and H. Kinoshita 1985. Secular perturbations of asteroids in secular resonances. Celest. Mech. 36, 391-407.

Poincaré, H. 1892. Les Méthodes nouvelles de la Mécanique Céleste. Gauthier Villars, Paris.

Šidlichovský, M. 1989. Secular resonances and the second fundamental model. Bull. Astron. Inst. Czech. 40, 92-104.

Sidlichovský, M. 1990. The existence of a chaotic region due to the overlap of secular resonances $\nu_{5}$ and $\nu_{6}$. Celest. Mech. 32, 177-196.

Scholl, H., Ch. Froeschlé, M. Yoshikawa, H. Kinoshita and G. H. Williams 1989. Secular Resonances. In Asteroids II ( R.P. Binzel, T.Gehrels, M.S. Matthews, Eds.), 845-861, Univ. of Arizona Press.

Scholl, H. and Ch. Froeschlé 1991. The $\nu_{6}$ Secular Resonance Region near 2.AU : a Possible Source of Meteorites. Astron. Astrophys. 245, 316-321.

Tisserand, M. F. 1882. Mémoire sur les mouvements séculaires des plans des orbites des trois planètes, Ann. Obs. Paris 16, E1-E57.

Wetherill, G. W. 1977. In Comets, Minor planets and Meteorites (A.H. Delsemme ,Ed.), 283-292, Univ. of Toledo Press.

Wetherill, G. W. 1979. Steady state populations of Apollo-Amor objects. Icarus 37, 96112.

Wetherill, G. W. 1988. Where do the apollo objects come from? Icarus 76, 1-18.

Wetherill, G. W. and J. G. Williams 1979. In Origin and Distribution of the Elements (L. H. Ahrens, Ed.), 19-31, Pergamon Oxford/ New York.

Williams, J. G. 1969. Secular perturbations in the Solar System. Ph.D. Dissertation, Univ. California Los Angeles.

Williams, J.G. and J. Faulkner 1981. The positions of secular resonances surfaces. Icarus 46, 390-399.

Yoshikawa, M. 1987. A simple analytical model for the secular resonance $\nu_{6}$ in the asteroidal belt. Celest. Mech. 40, 233-272. 